\title{
Anisotropic behavior of water in ferroelectric liquid crystals
}

\author{
G. Singh, ${ }^{1}$ A. Choudhary, ${ }^{2}$ G. Vijaya Prakash, ${ }^{1, *}$ and A. M. Biradar ${ }^{2, \dagger}$ \\ ${ }^{1}$ Nanophotonics Laboratory, Department of Physics, Indian Institute of Technology, New Delhi 110016, India \\ ${ }^{2}$ Liquid Crystal Group, National Physical Laboratory, Dr. K. S. Krishnan Road, New Delhi 110012, India
}

\begin{abstract}
The outcome of water addition in ferroelectric liquid crystal (FLC) has been investigated in uniform and defect-free homogeneous and homeotropically aligned monodomain sample cells from electro-optical and dielectric spectroscopic measurements. The lagging in optical response between nonconducting (spatially variable switching) and conducting (conventional switching) portions of water added FLC sample cell has been observed by frequency-dependent electro-optical studies. The bias-dependent water related new relaxation peak near the conventional Goldstone mode relaxation process has been observed only in the homogeneous alignment and not in the homeotropic one. Further, the significant increment in dielectric anisotropy as well as faster diffusion of water along long molecular axis than short molecular axis has also been monitored. These studies strongly suggest that the distribution of water is anisotropic in FLC medium and could be the reason for new relaxation peak in the water added FLC sample.
\end{abstract}

\section{INTRODUCTION}

A two-dimensional complex fluid, i.e., liquid crystal (LC), with translational ordering of molecules that leads to the formation of layers is known as smectic phase. The tilted geometry of molecular director in these layers entitled the phase as smectic- $C(\mathrm{Sm}-C)$ phase, in which the addition of an asymmetric carbon molecule generates the helicoidal structure of molecular director and hence the generation of ferroelectricity in liquid crystal and termed as ferroelectric liquid crystal (FLC) [1,2]. It has been found that addition of isotropic compounds [3-6] and nanoparticles [7-11] to these liquid crystal materials can be utilized to alter their basic properties. Therefore, to understand the behavior of interaction of isotropic compounds such as water with LC, several studies at the interface of these compounds have been carried out to analyze the roughening, wetting, and layering at the interface of two liquids [12-16].

The interface phenomenon of water and nematic liquid crystal (NLC) has shown that the increase in the concentration of surfactant in the water leads to the freezing of NLC material at the interface $[17,18]$. The excess concentration of surfactant in water produces a new phase at the interface of water and smectic liquid crystal, while lower concentration of surfactant produces the triple point above the smectic- $A$ $(\mathrm{Sm}-A)$ to isotropic (Iso.) transition temperature $\left(T_{c}\right)$ [13]. The interface study of smectic liquid crystal and water (nonionic) shows the complete and incomplete wetting, which is a matter of finite and infinite numbers of smectic layer transition [19]. Thus, at the interface of water and liquid crystal, the intermolecular interactions are affected.

The most studied compound is water, which distributes itself when mixed with system into two basic categories: (i) free water and (ii) bound water. As an example of biological system, the biological free water shows its natural relaxation frequency while bound water (produces hydration shell of water) shows a different dielectric relaxation frequency due to the coupling of $\mathrm{H}_{2} \mathrm{O}$ molecules with the system in which it is embedded [20-25]. Similarly, the bound water in soil matrix also shows a different dielectric relaxation frequency as compared to the free water due to the action of binding forces on water molecules [26].

The monolayer of FLC compounds with chiral groups on water-glycerol mixture surface allows the autorotation of FLC molecules [27]. However, in some FLC compounds it has been observed that the chiral groups of FLC in water does not favor the autorotation of FLC molecules i.e., the nonchiral end and not the chiral end part of FLC molecule should be attached with water surface [28]. Recently, we demonstrated that water mixed FLC introduces the spatially variable switching in nonelectrode region (near to the electrode edge) of the sample cell [29]. We have interpreted the results that the presence of higher amount of water in between two smectic layers [30] reduces surface tension between these layers and the curved electric field enabled to switch the molecules outside the electrode projection.

In this paper, we report changes in the properties of FLC (such as switching and dielectric permittivity) in water added FLC system through electro-optical and dielectric spectroscopic investigations. In the present study, bias-dependent water related new relaxation peak, increment in the dielectric anisotropy, and faster diffusion of water along the long molecular axis strongly suggest that the distribution of water in FLC medium is anisotropic.

\section{EXPERIMENTAL DETAILS}

The sample cells have been fabricated by assembling indium tin oxide (ITO) coated borosilicate glass plates having $\lambda / 2$ flatness. The square patterns of ITO $(\sim 10 \Omega / \square)$ have been prepared by photolithography technique, which act as electrodes to apply an electric field to the FLC sample. These substrates have been treated with adhesion promoter (phenyl tricholorosilane in toluene) followed by coating of nylon 6/6. 
The rubbing of nylon coated substrates has been done by a buffing machine (LCBM, Irwin, USA) using velvet cloth wrapped on a drum roller under the fixed rubbing parameters (rubbing strength $R_{S}=N M(2 \pi r n / v-1)$, where $N$ is the number of strokes with which the substrate has been rubbed, $M$ is the depth of fibers of the fabric deformed due to pressed contact, $r$ is the radius of the drum, $n$ is the rotational speed of drum, and $v$ is the translational speed of the substrate $\left[R_{S}=14.837 \mathrm{~m}\right.$ when $N=2, M=1.038 \mathrm{~mm}$, $r=39.8 \mathrm{~mm}, n=2000 \mathrm{rpm}$, and $v=7 \mathrm{~mm} / \mathrm{s}]$ ). A uniform thickness of around 3.5-6.5 $\mu \mathrm{m}$ has been maintained between the two patterned plates by using Mylar spacers of same thickness. In the present work, most of the electrooptical and dielectric investigations have been carried out in homogeneous aligned FLC cells, where the alignment has been achieved through above-mentioned rubbing technique. However, homogeneous and homeotropic alignments for dielectric anisotropic measurements have been obtained by applying a magnetic field $(1.2 \mathrm{~T})$ to the FLC cell. The homogeneous to homeotropic or vice versa alignment has been converted only by changing the direction of applied magnetic field to the cell [32]. The uniform and defect-free alignments (homogeneous and homeotropic) of present FLC mixture have been ensured by high-resolution optical polarizing microscopy (HROPM). The phase sequence of the studied FLC mixture (CS1026, Chisso Corporation, Japan) having a spontaneous polarization value of $26 \mathrm{nC} / \mathrm{cm}^{2}$ and a pitch value of $10 \mu \mathrm{m}$ is as follows:

$$
\begin{gathered}
-7{ }^{\circ} \mathrm{C} \quad{ }^{6{ }^{\circ} \mathrm{C}} \quad 82^{\circ} \mathrm{C} \quad 91^{\circ} \mathrm{C} \\
\text { crystal } \leftrightarrow \mathrm{Sm}-C^{*} \leftrightarrow \mathrm{Sm}-A^{*} \leftrightarrow \mathrm{N} \leftrightarrow \text { isotropic }[\mathrm{CS} 1026] .
\end{gathered}
$$

The methodology utilized here for water addition into the FLC mixture and filling it into the sample cell has been followed as described in our previous report [29]. Electrooptical measurements have been carried out by using HROPM (Axioscope-40, Carl Zeiss, Germany), where the photodiode has been mounted on the microscope to record the optical transmission response of FLC sample cell. Dielectric spectroscopic measurements have been recorded by using impedance analyzer (Wayne Kerr 6450A) in the frequency range of $20 \mathrm{~Hz}$ to $1 \mathrm{MHz}$. A temperature controller (Julabo F25) has also been used to maintain the temperature stability of FLC sample within the accuracy of $\pm 0.01{ }^{\circ} \mathrm{C}$.

\section{RESULTS AND DISCUSSIONS}

\section{A. Electro-optical studies}

The room-temperature optical micrographs of water added and pure FLC samples have been presented (Fig. 1). Figure 1(a) shows the texture of conducting and nonconducting portions of water added FLC sample under crossed polarizing microscope. At $0 \mathrm{~V}$ bias, there is no observable distinction in texture of both portions of the sample cell. The natural switching phenomenon of FLC in the conducting portion occurs at small de voltage with a magnitude of $1 \mathrm{~V}$ [Fig. 1(b)], due to which the change in birefringence of textures can also be seen clearly. However, interesting observation is that a minute change starts to occur in the texture of nonconducting portion at the edge of electrode. When the magnitude
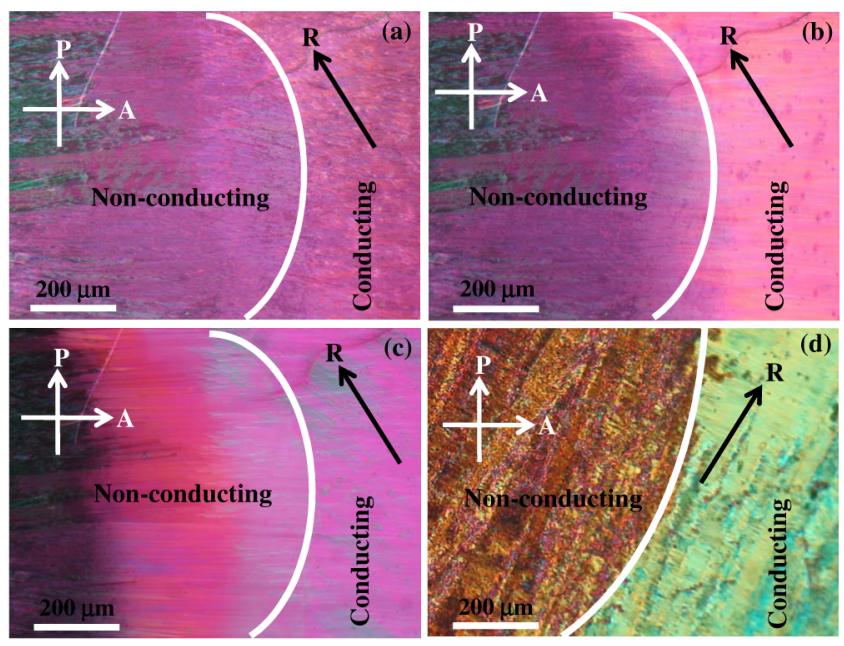

FIG. 1. (Color online) Room-temperature optical micrographs of water added FLC sample under crossed polarizing system at (a) 0 , (b) 1 , and (c) $20 \mathrm{~V}$, and in pure FLC sample at (d) $20 \mathrm{~V}$. Scale bar: $200 \mu \mathrm{m}$. The notations "Conducting" and "Non-conducting" have been used for the electrode region as well as nonelectrode region of sample cell, respectively. The rubbing direction $(R)$ with respect to crossed polarizing system has been shown by single black arrow.

of applied dc voltage is increased to $20 \mathrm{~V}$ then the area of spatially variable switching in the nonconducting portion of the sample cell also increases to its greater extent. Since this change is spatially variable starting from the conducting to nonconducting portion with significant variation in the optical tilt angle of sample cell [29], therefore, the coverage area of such gradient switching in nonconducting portion increases as we increase voltage at conducting portion of the sample cell [Fig. 1(c)]. However, no traces of switching with or without spatial variation in the pure FLC sample have been observed in the nonconducting portion of the cell [Fig. 1(d)], unlike in water added FLC sample.

To explore more about such a fascinating switching phenomenon, the frequency-dependent electro-optical studies have also been performed individually in both conducting and nonconducting portions of water added FLC sample cell (Figs. 2 and 3) by using a photodiode mounted on optical polarizing microscope. Figure 2 shows the frequencydependent optical response in the conducting portion of sample cell to the applied time delayed square pulse. It has been observed that at low frequency $(500 \mathrm{mHz})$ switching of FLC molecules follows the applied pulse [Fig. 2(a)] but shows no traces of memory effect, because there is a sharp drop in the optical transmission as soon as the pulse reaches the $0 \mathrm{~V}$ level. When the frequency of the applied pulse is increased to $2 \mathrm{~Hz}$ then no significant delay in the optical response as well as memory effect has been observed. On further increasing the frequency of applied pulse to $8 \mathrm{~Hz}$, a little stability has been observed without any delay in optical response to the applied pulse. Thus, we can infer that the studied material is responding to the applied pulse even at higher frequencies as well.

Figure 3 shows the optical response in the nonconducting portion of frequency-dependent applied time delayed square 


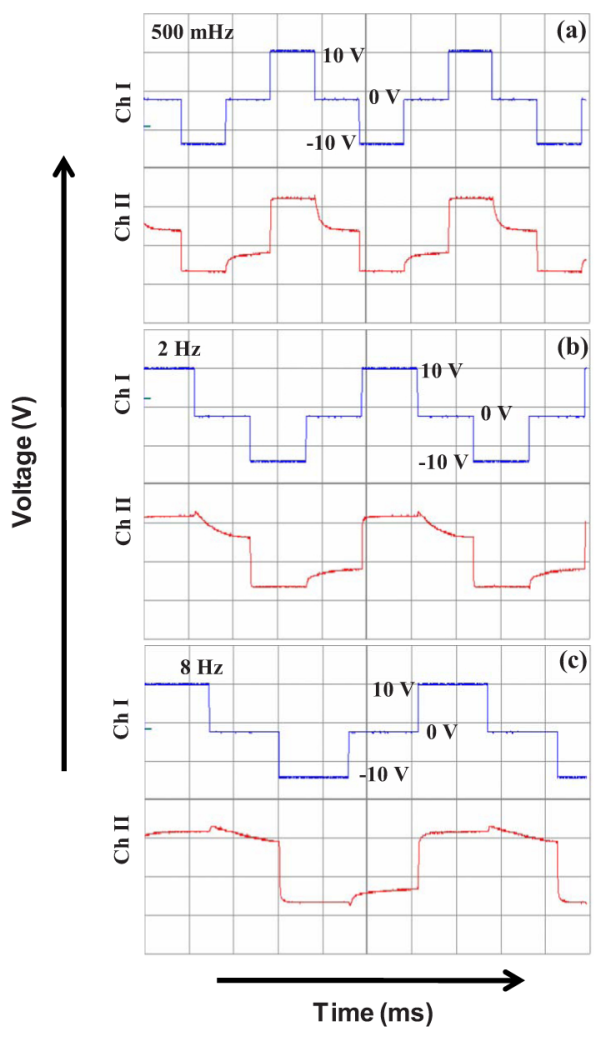

FIG. 2. (Color online) Optical response as a function of frequency of applied time delayed square pulse in the conducting portion of water added FLC sample cell at (a) $500 \mathrm{mHz}$, (b) $2 \mathrm{~Hz}$, and (c) $8 \mathrm{~Hz}$, where $\mathrm{Ch}$ I and $\mathrm{Ch}$ II show driving square voltage pulse and its optical response, respectively. The time scale per division (div) $(10 \mathrm{~ms} / \mathrm{div})$ is on the $x$ axis. The voltage on the $y$ axis for channels $\mathrm{Ch}$ I and $\mathrm{Ch}$ II are 08.00 and $0.02 \mathrm{~V} / \mathrm{div}$, respectively.

pulse in the conducting portion of water added FLC sample cell, where spatially variable switching occurs near the electrode edge (at $\sim 6 \mu \mathrm{m}$ away from the electrode). Figure 3(a) shows that there is observable delay in the optical switching response to the applied pulse at $500 \mathrm{mHz}$ and this switched state remains stable for short duration at $0 \mathrm{~V}$ level as compared to its counterpart in the conducting portion [Fig. 2(a)]. At $2 \mathrm{~Hz}$ frequency of the applied pulse [Fig. 3(b)], the delay in optical response further increases with increased stability. However, the sharpness in response degrades because the edges are not sharp in optical response pulse i.e., molecules are not too quick to switch with the applied pulse. At $8 \mathrm{~Hz}$ frequency, the optical response further delays with more degradation in the shape and amplitude of switching response due to the nonresponding nature of FLC molecules at high frequencies [Fig. 3(c)]. Overall the textural appearance and electro-optical studies indicate some different type of switching phenomenon happening in the nonconducting portion of the water added FLC sample cell unlike the switching in conducting portion of pure FLC sample cell.

\section{B. Dielectric studies}

The important and conclusive results have been reflected in the dielectric studies of water added FLC sample. The

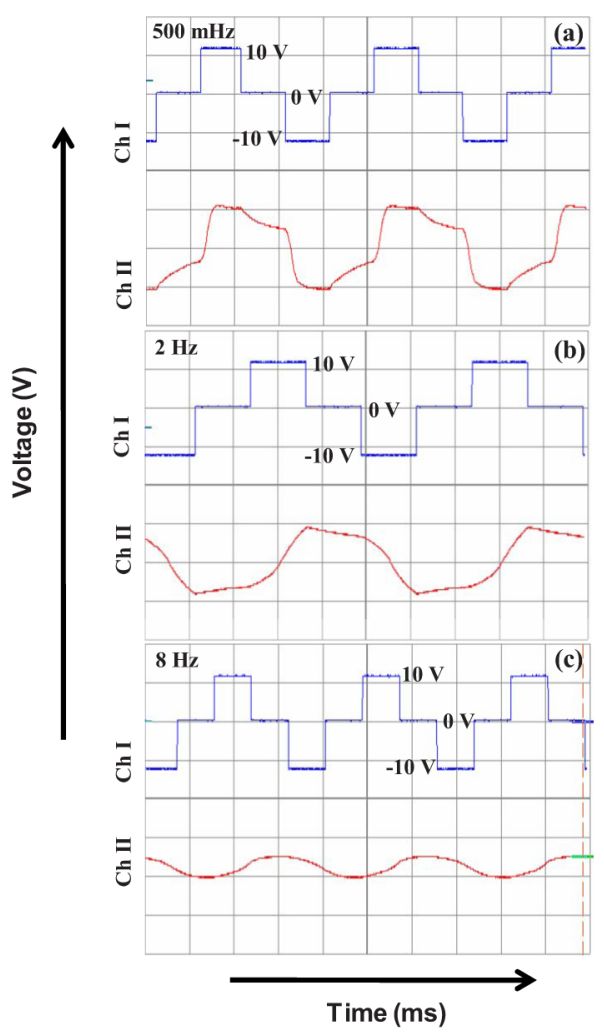

FIG. 3. (Color online) Optical response as a function of frequency of applied time delayed square pulse in the nonconducting portion of water added FLC sample cell at (a) $500 \mathrm{mHz}$, (b) $2 \mathrm{~Hz}$, and (c) $8 \mathrm{~Hz}$, where $\mathrm{Ch} \mathrm{I}$ and $\mathrm{Ch}$ II show driving square voltage pulse and its optical response, respectively. The time scale per division $(10 \mathrm{~ms} / \mathrm{div})$ is on the $x$ axis. The voltage on the $y$ axis for channels $\mathrm{Ch}$ I and $\mathrm{Ch}$ II are 08.00 and $0.02 \mathrm{~V} / \mathrm{div}$, respectively.

preliminary distinction in the dielectric properties of pure and water added FLC samples at room temperature has been presented (Fig. 4). The prime difference occurs at frequency lower than the Goldstone mode relaxation frequency in the homogeneous alignment. The dielectric permittivity $\left(\varepsilon^{\prime}\right)$ increases after addition of water into the FLC matrix [Fig. 4(a)]. This increment in the dielectric permittivity should also result into the increase in the refractive index of water added FLC sample than pure FLC one. Therefore, the change in the refractive index $(n)$ of FLC in the homogeneous alignment has been calculated by using the relation $n=\sqrt{\varepsilon_{\infty} / 1.05}$, where $\varepsilon_{\infty}$ shows dielectric permittivity $\left(\varepsilon^{\prime}\right)$ value at higher frequency, where it almost becomes independent of frequency [31]. The change in the refractive index of pure FLC with respect to the water added FLC has been given by $\delta n$ $=n_{\text {water }+F L C}-n_{F L C}=0.1409$, where $n_{\text {water }+F L C}=1.7167$ and $n_{F L C}=1.5759$ have been calculated at the frequency of $216.52 \mathrm{kHz}$ [Fig. 4(a)]. It is obvious here that the refractive index is higher in water added FLC sample than pure FLC one. Further, the $\tan \delta\left(=\varepsilon^{\prime \prime} / \varepsilon^{\prime}\right)$ spectra show the conventional Goldstone mode peak at $2.452 \mathrm{kHz}$ and also a new relaxation peak at $916.82 \mathrm{~Hz}$ in the sample containing water [inset of Fig. 4(a)]. The observed new relaxation peak at $916.82 \mathrm{~Hz}$ has been found very sensitive to the applied dc bias fields. As seen in Fig. 4(b), the new relaxation peak 

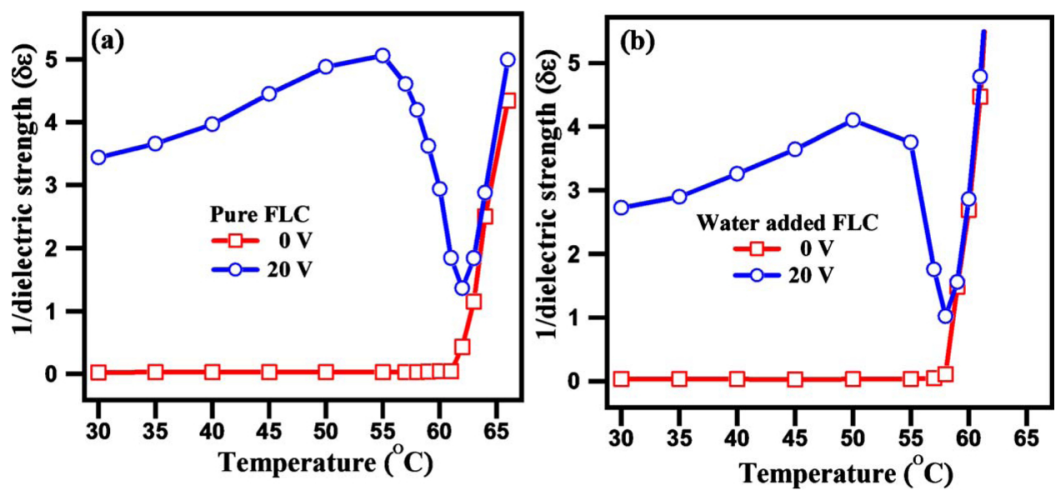

FIG. 7. (Color online) Temperature-dependent inverse of dielectric strength $(1 / \delta \varepsilon)$ behavior in homogeneously aligned (a) pure and (b) water added FLC samples at 0 and $20 \mathrm{~V}$ dc biases.

applied to remove the Goldstone mode contribution even in the homeotropic alignment. Therefore, increment in dielectric anisotropy of water added FLC sample indicates the anisotropic distribution of water in FLC sample.

The temperature-dependent behavior of the inverse of dielectric strength $\left[1 / \delta \varepsilon=1 /\left(\varepsilon_{0}-\varepsilon_{\infty}\right)\right]$ in the homogeneously aligned pure and water added FLC samples has been shown [Figs. 7(a) and 7(b)]. This study has been mainly carried out to observe the validity of the Curie-Weiss law in the analysis of ferroelectric phase and effect of water addition in the Sm- $C^{*}-\mathrm{Sm}-A^{*}$ phase transition temperature $\left(T_{c}\right)$ of water added FLC sample as compared to the pure FLC one. The curve at $0 \mathrm{~V}$ de bias in Figs. 7(a) and 7(b) demonstrates that $1 / \delta \varepsilon$ with respect to temperature remains constant due to the presence of Goldstone mode in $\mathrm{Sm}-C^{*}$ phase and it increases continuously in the $\mathrm{Sm}-A^{*}$ phase. In $\mathrm{Sm}-C^{*}$ phase near $T_{c}$, Goldstone mode becomes weak as intrinsic tilted structure of molecules disappears gradually and only induced tilted fluctuations appear which generates the soft mode. Thus, at $T_{c}$, Goldstone mode fully disappears and soft mode becomes dominant. The analysis of soft mode in $\mathrm{Sm}-C^{*}$ and $\mathrm{Sm}-A^{*}$ phases leads to examine the validity of the Curie-Weiss law. Therefore, it is essential to examine the contribution of soft mode and hence its respective dielectric strength in the Sm- $C^{*}$ phase. However, it is difficult to get soft mode near $T_{c}$. in $\mathrm{Sm}-C^{*}$ phase without suppressing Goldstone mode. Therefore, a high dc bias with a magnitude of $20 \mathrm{~V}$ has been applied to suppress the Goldstone mode near $T_{c}$ to observe the soft-mode presence in the Sm- $C^{*}$ phase. The curve at 20 $\mathrm{V}$ dc bias in Figs. 7(a) and 7(b) shows the decreasing behavior of the inverse of dielectric strength below $T_{c}$ while increasing behavior above $T_{c}$, which satisfies the validity condition of the Curie-Weiss law in the present case. Since the dip in $1 / \delta \varepsilon$ is always at $T_{c}$ of $\mathrm{Sm}-C^{*}-\mathrm{Sm}-A^{*}$ phase transition of FLC materials, therefore, the shift in $T_{c}$ could be analyzed by the comparison of $1 / \delta \varepsilon$ behavior in both pure and water added FLC samples. The dip at lower temperature [Fig. 7(b)] shows reduction of $4{ }^{\circ} \mathrm{C}$ in $T_{c}$ of $\mathrm{Sm}-C^{*}-\mathrm{Sm}-A^{*}$ phase transition in the water added FLC sample as compared to the pure FLC one [Fig. 7(a)]. This might be due to the reduction in the strength of electrostatic interlayer interaction in water added FLC sample.

The time-dependent study of diffusion of water from water added FLC side to pure FLC side has been analyzed in the same cell [Fig. 8(a)] having two patterned electrodes on a single substrate, where one is for pure FLC and the other is for water added FLC, thereby the effect due to different samplings could be avoided. Water added FLC material has been injected through the opening of one side and a pure FLC material through the opening of another side in this double patterned single cell at a temperature, which is just below the nematic to isotropic phase transition temperature. The trapped air will leak out through the side openings and both side fillings make an interface of two materials in the sample cell. In this way, both water added and pure FLC materials have been filled in the same cell. Figures 8(b) and 8(c) exhibit the appearance of peak due to water in the $\tan \delta$ vs frequency spectra with respect to time in the homogeneous aligned sample cell. Two types of samples having different directions of alignment with respect to the flow of water

(a)
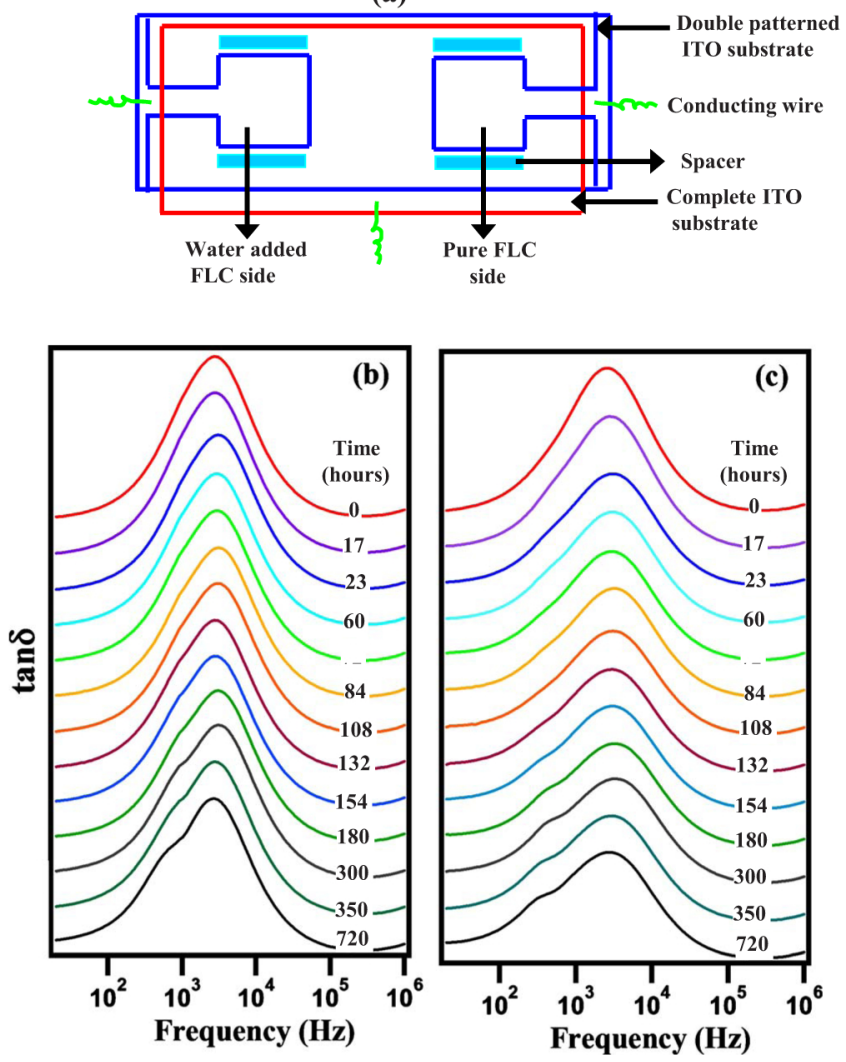

FIG. 8. (Color online) (a) Schematic of double patterned liquidcrystal cell and the time-dependent diffusion of water from water added FLC region to pure FLC region (b) parallel and (c) perpendicular to the long molecular axis of FLC molecule, respectively. 
have been used for the measurements. In the first type, the flow of water from water added side to pure FLC side is along the long molecular axis (i.e., perpendicular to the layers) [Fig. 8(b)], whereas it is along the short molecular axis (i.e., parallel to the layers) in the other type [Fig. 8(c)]. After filling both types of samples, the measurements have been performed at room temperature only in the pattern having pure FLC material to confirm whether water has diffused into it from water added FLC side or not. It exhibits the relaxation peak due to water near the Goldstone mode relaxation in the water added FLC side unlike Goldstone mode relaxation in the pure FLC side [Figs. 8(b) and 8(c)]. It has been observed that the water peak is clearly visible after 200 $\mathrm{h}$ in both types of sample cells. Water diffuses from water added FLC region to the pure FLC region of sample cell and binds with FLC molecules, which can be clearly seen in the form of a new relaxation peak in $\tan \delta$ versus frequency spectra [Figs. 8(b) and 8(c)]. The peak due to water starts appearing around $72 \mathrm{~h}$ (water diffusion along the long molecular axis) [Fig. 8(b)] and after $132 \mathrm{~h}$ (water diffusion along the short molecular axis) [Fig. 8(c)]. This nonuniform diffusion of water in FLC matrix is due to the anisotropic nature of FLC material and shows the nonuniform distribution of water.

Thus, the unique behavior of FLC material tailored by the water addition has been studied through electro-optical and dielectric spectroscopic measurements. The evidences of slower switching phenomenon in the nonconducting region than in the conducting region of water added FLC sample has been observed in their electro-optical investigations. The water related relaxation peak near the Goldstone mode is found to be highly bias dependent. The presence of this new relaxation peak only in the homogeneous alignment strongly suggests its association with a unique relaxation process, i.e., Goldstone mode. The significant increment in the dielectric anisotropy of water added FLC sample throughout the temperature range demonstrates that the distribution of water in FLC would be anisotropic. The time-dependent diffusion studies reveal that the diffusion of water is faster along the long molecular axis and slower along the short molecular axis of FLC molecule.

\section{CONCLUSION}

We have reported the anisotropic distribution of water in the FLC matrix through electro-optical and dielectric investigations. The effect of addition of water in the FLC matrix exhibits the switching phenomenon in the region outside of electrodes unlike switching in pure FLC. The electro-optical studies confirm the slow switching in the region outside the electrode projection region. The dielectric studies show that highly bias-dependent water relaxation peak appears only in the homogeneous alignment, which strongly suggests for its association with a unique relaxation process, i.e., Goldstone mode. The significant increment in the dielectric anisotropy has been observed in the water added FLC as compared to pure FLC. It has also been identified that the water diffusion is faster along the long molecular axis than the short molecular axis of FLC molecule. Moreover, these studies strongly suggest that the distribution of water is anisotropic in the FLC medium and could be the reason for new relaxation peak in the water added FLC sample.

\section{ACKNOWLEDGMENTS}

The authors sincerely thank Professor R. C. Budhani of the National Physical Laboratory for continuous encouragement and interest in this work. The authors (G.S. and A.C.) are thankful to Council of Scientific and Industrial Research (CSIR), New Delhi, India for financial assistance.
[1] J. W. Goodby, R. Blinc, N. A. Clark, S. T. Lagerwall, M. A. Osipov, S. A. Pikin, T. Sakurai, K. Yoshino, and B. Zeks, Ferroelectric Liquid Crystals: Principles, Properties and Applications (Gordon and Breach, New York, 1991).

[2] R. B. Meyer, L. Liebert, L. Strzelecki, and P. Keller, J. Phys. (Paris), Lett. 36, 69 (1975).

[3] P. E. Lammert, D. S. Rokhsar, and J. Toner, Phys. Rev. E 52, 1778 (1995).

[4] T. C. Lubensky, D. Pettey, N. Currier, and H. Stark, Phys. Rev. E 57, 610 (1998).

[5] P. Poulin and D. A. Weitz, Phys. Rev. E 57, 626 (1998).

[6] J. Yamamoto and H. Tanaka, Nature (London) 409, 321 (2001).

[7] P. Fabre, C. Casagrande, M. Veyssie, V. Cabuil, and R. Massart, Phys. Rev. Lett. 64, 539 (1990).

[8] O. V. Kuksenok, R. W. Ruhwandl, S. V. Shiyanovskii, and E. M. Terentjev, Phys. Rev. E 54, 5198 (1996).

[9] I. In, Y.-W. Jun, Y. J. Kim, and S. Y. Kim, Chem. Commun. (Cambridge) 2005, 800.

[10] S. Krishna Prasad, K. L. Sandya, G. G. Nair, U. S. Hirernath,
C. V. Yelamaggad, and S. Sampath, Liq. Cryst. 33, 1121 (2006).

[11] H. Qi and T. Hegmann, J. Mater. Chem. 16, 4197 (2006).

[12] G. Forgacs, R. Lipowsky, and Th. M. Nieuwenhuizen, In Phase Transition and Critical Phenomenon (Academic, London, 1991).

[13] Ch. Bahr, Phys. Rev. Lett. 99, 057801 (2007), and references therein.

[14] G. P. Crawford, R. J. Ondris-Crawford, J. W. Doane, and S. Zumer, Phys. Rev. E 53, 3647 (1996).

[15] B. M. Ocko, A. Braslau, P. S. Pershan, J. Als-Nielsen, and M. Deutsch, Phys. Rev. Lett. 57, 94 (1986).

[16] G. J. Kellogg, P. S. Pershan, E. H. Kawamoto, W. F. Foster, M. Deutsch, and B. M. Ocko, Phys. Rev. E 51, 4709 (1995).

[17] J. M. Brake, A. D. Mezera, and N. L. Abbott, Langmuir 19, 6436 (2003).

[18] A. D. Price and D. K. Schwartz, J. Phys. Chem. B 111, 1007 (2007).

[19] A. M. Somoza, L. Mederos, and D. E. Sullivan, Phys. Rev. Lett. 72, 3674 (1994). 
[20] R. Pethig, Annu. Rev. Phys. Chem. 43, 177 (1992).

[21] J. A. Rupley and G. Careri, Adv. Protein Chem. 41, 37 (1991).

[22] I. D. Kuntz, Jr. and W. Kauzmann, Adv. Protein Chem. 28, 239 (1974).

[23] W. Saenger, Annu. Rev. Biophys. Biophys. Chem. 16, 93 (1987)

[24] M. M. Teeter, Annu. Rev. Biophys. Biophys. Chem. 20, 577 (1991).

[25] N. Nandi and B. Bagchi, J. Phys. Chem. B 101, 10954 (1997).

[26] M. A. Hilhorst, C. Dirksen, F. W. H. Kampers, and R. A. Feddes, Soil Sci. Soc. Am. J. 65, 311 (2001).

[27] Y. Tabe and H. Yokoyama, Nat. Mater. 2, 806 (2003).
[28] P. M. Milczarczyk-Piwowarczyk, A. Zywociński, K. Noworyta, and R. Hołyst, Langmuir 24, 12354 (2008).

[29] A. Choudhary, I. Coondoo, J. Prakash, K. Sreenivas, and A. M. Biradar, Appl. Phys. Lett. 94, 174101 (2009).

[30] F. D. Blum, A. S. Padmanabhan, and R. Mohebbi, Langmuir 1, 127 (1985).

[31] W. Haase and S. Wrobel, Relaxation Phenomena: Liquid Crystal, Magnetic Systems, Polymers, High- $T_{c}$ Superconductors, Metallic Glasses (Springer, Berlin, 2003).

[32] G. Singh, G. Vijaya Prakash, S. Kaur, A. Choudhary, and A. M. Biradar, Physica B 403, 3316 (2008). 